\title{
Study on the overlooking Interface of Mountain City skyline based on Fractal Theory
}

\author{
Liang Zhao ${ }^{1}$, Guanwen Ding, ${ }^{1, *}$, Yanan Dong ${ }^{2}$ and Yue $\mathrm{Wu}^{1}$ \\ ${ }^{1}$ School of Architectural Planning, Shandong Jian Zhu University \\ ${ }^{2}$ School of Chemical Engineering, University of Jinan \\ ${ }^{*}$ Corresponding author
}

\begin{abstract}
Based on the characteristics of fractal geometry theory and the reflection of Foshan in Jinan as a wedge, this paper quantitatively describes the shape of the overlooking interface of Foshan by calculating the fractal dimension of the overlooking interface. Combined with the city skyline dimension of big cities at home and abroad, this paper sums up the variation law of fractal dimension of mountain city skyline overlooking interface from many aspects, and finally starts from the angle of fractal theory. The evaluation system of mountain city skyline and the idea of planning control in the future are put forward.
\end{abstract}

Keywords-component; fractal geometry; Foshan reflection; mountain overlooking interface; fractal dimension

\section{INTRODUCTION}

With the rapid development of urban economy, more and more high-rise buildings are built up in the city, which results in the destruction of the original city skyline or natural skyline.In this case, the destruction is especially prominent in mountainous cities. With the appearance of high-rise buildings, it blocks the natural mountains, resulting in a serious impact on the skyline of the mountains in the city background.It is an urgent problem in mountain town planning. How to regain the "mountain city" space environment and create a beautiful mountain city space pattern is the focus of the future mountain city space development and construction.

\section{FRACTAL GEOMETRY THEORY}

\section{A. Introduction to Fractal Geometry Theory}

Fractal geometry is proposed by mathematician Munderboro to describe the relatively complex and unordered geometric bodies in nature which cannot be expressed by traditional Euclidean geometry such as mountain ridge river coastline and so on.Its most basic feature is that fractal images with complex geometry are represented by fractal dimension and mathematical method.Using its most prominent essential feature, self-similarity, the rules of urban skyline can be expressed, irregular and even fragmented geometric objects.

In recent years, in the process of development of construction industry in our country, people gradually realize the importance of fractal science, so it is widely used in architectural design.In this way, it not only satisfies the individualized characteristics of modern architectural design, but also fully reflects the artistic beauty of architectural design. Fractal geometry provides a suitable geometric description method for irregular shapes in nature.With the aid of computer, architects consciously use fractal geometry knowledge to generate more abundant space.Maintaining the Integrity of the Specifications

\section{B. Fractal Dimension Calculation Method}

Fractal dimension, as thebasic measure of shape description of irregular geometric bodies, can describe the classification characteristics of geometric bodies because it jumps out of the spatial dimensions of one dimension, two dimensions and three dimensions, and approaches to the authenticity of complex systems indefinitely.Measure irregular geometry.

$$
D=\frac{\ln b}{\ln a}
$$

$\mathrm{D}$ is the fractal dimension of the geometric object $\mathrm{A}$ is the magnification of the geometric object and $\mathrm{b}$ is the original volume multiple.

The fractal dimension reflects the filling ability of the graph to space and the complexity and irregularity of the boundary.For planar portraits, the dimension value is generally between 1 / 2 and the larger the value is, the more complex the figure is. Usually, 1.5 is taken as the boundary, the value less than 1.5 indicates that the figure is relatively simple, whereas the figure larger than 1.5 indicates that the figure tends to be complex.

This paper deals with the calculation and analysis of fractal dimension by the method of calculating fractal dimension , such as area - perimeter correlation method, half - variance method, and box dimension method. With the change of $r$, the value of $\mathrm{Nu} r$ will also change. The smaller the size, the larger the value, and vice versa.At last, a series of $r$ and Nrn are fitted in the double logarithmic coordinates by the least square method. The slope of the obtained line is called the fractal dimension D.

$$
D=-\lim _{r \rightarrow 0} \frac{\log N(\mathrm{r})}{\log r}
$$




\section{FRACTAL CHARACTERISTICS AND FRACTAL DIMENSION INFLUENCING FACTORS OF MOUNTAIN CITY OVERLOOKING INTERFACE}

The urban view interface mainly refers to the landscape interface formed by the horizontal plane in which all the scenery within its visual range is perpendicular to its view when the person in the city overlooks a distant mountain range. The main components of the overlooking interface of mountainous cities are the mountains and skylines of the cities. The city skyline is the most important factor affecting the view interface of the mountain city, and it is also the most difficult factor to control compared to the mountain body of the city.

\section{A. Fractal Features of the Overlooking Interface}

Urban skyline is a contour line formed by a building or a group of buildings and other objects in the background of the sky. It is a two-dimensional projection of the superposition of three-dimensional urban form. The skyline has obvious fractal characteristics, and the same skyline has similar self-similar fine structure at different scales.At the same time, its dimension is strictly larger than its topological dimension (the topological dimension of line is $1 \sim 1$, and the value is between 1 and 2 ). The higher correlation coefficient in the subsequent skyline dimension calculation data further proves that it has remarkable fractal characteristics.

The urban skyline is divided into three categories, one is the straight urban skyline, the second is the urban skyline with center, and the third is the undulating skyline.

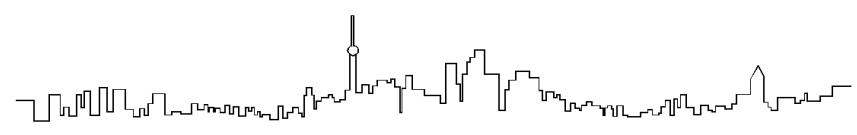

Shanghai skyline

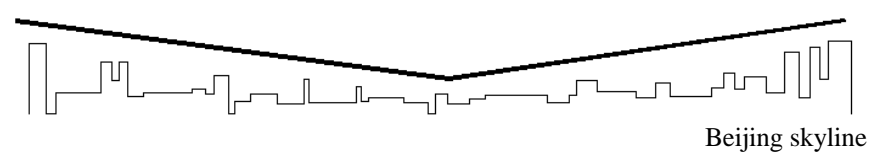

The undulating contours of natural mountains are not only the grand background of urban space, but also have a good relationship with urban buildings on the plane, and rise and fall on the facade with the skyline of urban architecture.Form a natural landscape pattern and urban construction elements of the coordinated scene.

\section{B. Overlooking Interface Influencing Factors}

The urban skyline is the main factor that affects the view interface of mountain city. If we control the city skyline well, we can form a good view of the city and bring a good visual experience to the city residents.There are many factors affecting the urban skyline, summarized as the following.

1) Historical factors. The city skyline is not a static contour and scene, it records the mark of each historical period of a certain place, and fully reflects the social outlook and the level of economic development of each period of the city.The history of urban construction has gone through such a long process, all aspects of the urban skyline changes have traces of historical change.As the city skyline changes, its fractal dimension is updated dynamically.

2) Urban construction factors. As the object of urban residents' view, the view interface of mountain city is the relationship between mountain body and architectural community on the elevation.Under the influence of building density, height, open intensity and the combination of buildings, different urban construction forms will be formed in different functional plots in cities, so various fractal dimensions will be formed.

3) Population factors. Overlooking is the behavior of city residents, and the view interface of mountain city is the subjective cognition of city residents.Urban residents' perception of the overlooking interface is influenced by many factors, such as view point, visual angle, visual distance and so on. None of these factors will affect the fractal dimension of the overlooking interface.

\section{STUDY ON THE OVERLOOKING INTERFACE AND FRACTAL OF JINAN MOUNTAIN CITY}

\section{A. Ji'nan Introduction and the Origin of Foshan Reflection}

As a famous historical and cultural city of the country, Jinan, "Mountain Spring Lake River City" is the characteristic element of its urban space, and the mountain plays an important role in the urban space structure. "Foshan reflection, Qiyan Nine points,"The landscape of magpie and rain reflects the spatial scene of the early Jinan mountain city, the mountain shape and the urban construction are intertwined, and the spatial relationship structure between the city and the mountain plays an important role in the development of the city.

Jinan famous scenery "Foshan reflection", when the weather is clear from the northern shore of Daming Lake to the south view, Qianfoshan completely reflected in the Daming Lake, mountains, lakes, shadow integrated.But as Jinan develops and builds, Daming Lake faces many challenges as it expands into Jinan: rising building heights, increasingly fragmented urban skylines,More and more disorderly buildings near the lake are eroding the pleasant spatial scale of Daming Lake, and it is becoming more and more difficult to see the reflection of Foshan.
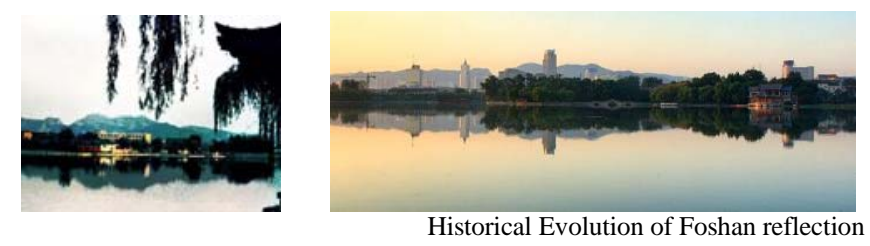

\section{B. Fractal Features of Foshan Reflection Overlooking Interface}

The observation point of Foshan reflection is Canglang Pavilion in Daming Lake Park, with a stone tablet engraved with four characters of Foshan reflection.Here the field of vision is more open, visual effect is significant, so, Canglang Pavilion as the view, overlooking the mountains, forming the whole mountain city overlooking interface. 


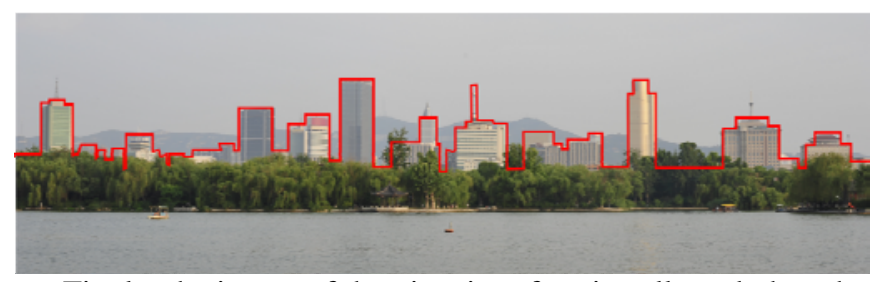

Firstly, the image of the view interface is collected, then the city skyline is described. According to the formula of fractal dimension $D=-\lim _{r \rightarrow 0} \frac{\log N(\mathrm{r})}{\log r}$, the fractal dimension $\mathrm{D}$ is 1.137.

\section{CONCLUSION}

After finishing the research and application of fractal theory at home and abroad, the calculation of fractal geometry and fractal dimension can effectively realize the quantitative evaluation of mountain city overlooking interface, and establish a good evaluation system of mountain city skyline based on fractal theory.It provides a powerful demonstration tool for the planning practice of beautifying the view interface of mountain city.

\section{A. How to Monitor and Evaluate the Overlooking Interface of Mountain City}

By taking the public space with wide vision and dense crowd as the view point, the urban skyline images observed from the view point are collected, and the relevant fractal dimension is calculated to evaluate the aesthetic degree of the overlooking interface.

\section{B. How to Realize the Planning and Control of Mountain City Overlooking Interface}

The planning control of the mountain city overlooking interface is based on the original development intensity, building height and so on. At the same time, the quantitative evaluation system of fractal dimension is added to realize the hierarchical control of the mountain city overlooking interface planning from macro to micro. Macroscopically, maintain the original urban form, through the accurate construction of fractal dimension, systematically link the relationship between the whole and the local of the city, realize the control of the whole skyline of the city; the mesoscopic level, Focus on the urban skyline of the central region, adopt appropriate fractal dimensions in the central area and other regions, respectively, in order to form a rich, vision-oriented view interface of mountain cities; microcosmic aspects, The fractal dimension of the overlooking interface is refined, and through the intervention of the fractal theory evaluation system, the building height, development intensity and even the exterior facade of the buildings in some parts of the city are repaired, and the three levels of management and control are progressive. Further make the image of the city more colorful.

\section{ACKNOWLEDGMENT}

This paper is funded by the National Natural Science Foundation of China (Project Grant No.51608309);

Research Project of Social Sciences Planning in Shandong Province ( Project Approval No. 17CWYJ21 )
Jinan City philosophy and Social Science Planning Project (Project Grant No.JNSK17B05)

\section{REFERENCES}

[1] Hong Kong Planning Departtment.Urban design guidelines[S].//Hong Kong planning standards and guidelines.2006.

[2] NIU Xinyi,LI Kaike, "Quantitative Analysis of Urban skyline based on Visual impact”,vol.3,Urban Planning Forum,2013,pp.99-105.

[3] YANG P R,PUTRA S,LI W.viewsphere:a Gis-based 3D visibility analysis for urban design enaluaton[J].Environment and Planning B;Planning and Design,2007,34(6);971-992.

[4] Cao Yingchun, Wang Yukun, Quantitative Analysis of Urban skyline based on Fractal Theory [J]. Urban problem. 2013,12,pp32-36.

[5] Wang Xing,Simulation algorithm of skyline contour of $\mathrm{V}$ street view based on AutoCAD VBA platform [J]. Chinese dwelling.6,213-214. 